\title{
A stochastic approach to grain surface chemical kinetics
}

\author{
N. J. B. Green ${ }^{1}$, T. Toniazzo ${ }^{2}$, M. J. Pilling ${ }^{3}$, D. P. Ruffle ${ }^{3}$, N. Bell ${ }^{3}$, and T. W. Hartquist ${ }^{2}$ \\ 1 Chemistry Department, Kings College London, London WC2R 2LS, UK \\ 2 Department of Physics and Astronomy, University of Leeds, Leeds LS2 9JT, UK \\ 3 School of Chemistry, University of Leeds, Leeds LS2 9JT, UK
}

Received 12 October 2000 / Accepted 2 July 2001

\begin{abstract}
A stochastic model of grain surface chemistry, based on a master equation description of the probability distributions of reactive species on grains, is developed. For an important range of conditions, rates of molecule formation are limited by low accretion rates, so that the probability that a grain contains more than one reactive atom or molecule is small. We derive simple approximate expressions for these circumstances, and explore their validity through comparison with numerical solutions of the master equation for $\mathrm{H}, \mathrm{O}$ and $\mathrm{H}, \mathrm{N}$, $\mathrm{O}$ reaction systems. A more detailed analysis of the range of validity of several analytic approximations and numerical solutions, based on exact analytical results for a model in which $\mathrm{H}$ and $\mathrm{H}_{2}$ are the only species, is also made. Though the use of our simple approximate expressions is computationally efficient, the solution of the master equation under the assumption that no grain contains more than two particles of each species usually gives more accurate results in the parameter regimes where the deterministic rate equation approach is inappropriate. The implementation of sparse matrix inversion techniques makes the use of such a truncated master equation solution method feasible for considerably more complicated surface chemistries than the ones we have examined here.
\end{abstract}

Key words. astrochemistry - molecular processes - methods: analytical - ISM: clouds - dust, extinction - ISM: molecules

\section{Introduction}

Allen \& Robinson (1977) developed the first interstellar gas-grain model wherein the surface chemistry is limited by the accretion rates of gas-phase species adsorbing on to the surface. They assumed that (i) all products of surface reactions are immediately injected into the gas-phase, and (ii) once two reactive species are adsorbed on the surface they will react before a third species arrives. Tielens \& Hagen (1982) extended the model of Allen \& Robinson to allow grain mantle accumulation; a Monte Carlo approach was employed to calculate the steady-state surface concentrations. The Allen \& Robinson (1977) and Tielens \& Hagen (1982) models represent the first attempts to apply a stochastic approach to the modelling of grain surface kinetics. Monte Carlo techniques to model the surface chemistry in interstellar clouds were subsequently employed by a number of authors (e.g. Tielens \& Allamandola 1987; Tielens 1995; Charnley et al. 1997; Tielens \& Charnley 1997). Charnley (1998) has employed a Monte Carlo technique to solve the master equation governing a model of interstellar gas phase chemistry and first suggested that a master equation approach can be applied to the problems of grain surface chemistry and to the coupling of the gas phase and grain surface chemistries in

Send offprint requests to: T. W. Hartquist, e-mail: twh@ast.leads.ac.uk interstellar clouds. If a grain surface is expected to contain few reactive species, such an approach to the treatment of the surface chemistry provides more accurate results than the deterministic rate equation method (Ruffle \& Herbst 2000). Owing to the computational expense of Monte Carlo methods, and the fact that, to date, it has not been possible to couple such an approach with a fully time-dependent gas-phase chemistry, Caselli et al. (1998) and Shalabiea et al. (1998) have introduced and employed modifications to the deterministic rate equations in an attempt to generalize such models so that they can be applied validly to problems in which the surface chemistry is accretion limited.

Here, we address the problem using a master equation approach to describe the probabilities that discrete numbers of atoms are adsorbed on a grain. In Sect. 2 an exact analytic solution is derived for the rate of $\mathrm{H}_{2}$ formation in a system in which $\mathrm{H}$ and $\mathrm{H}_{2}$ are the only species. The approach is then extended in Sect. 3 to the more complex systems addressed by Caselli et al., where we employ a numerical solution. We also make use of the fact that for a wide variety of interstellar environments the probability that a grain has more than one reactive atom or molecule on its surface is small. We introduce an approximation that yields analytic solutions that can readily be incorporated in numerical integrations. The approximations are compared with results from a virtually exact treatment 
of the simple $\mathrm{H}, \mathrm{H}_{2}, \mathrm{O}, \mathrm{OH}$, and $\mathrm{O}_{2}$ problem addressed by Caselli et al. In Sect. 4 we apply approximations to a bigger system, which also contains $\mathrm{H}_{2} \mathrm{O}, \mathrm{N}, \mathrm{NH}, \mathrm{NH}_{2}$, $\mathrm{NH}_{3}$, and $\mathrm{NO}$, as considered by Caselli et al. Though we have found that the simple approaches that we used to obtain results for Sects. 3 and 4 work well for the parameter regimes explored by Caselli et al., they are not valid for all parts of parameter space. To gain a better idea of their ranges of validity and to explore the utility of simple approximations, we investigate in Sect. 5 the errors introduced by several approximations for the $\mathrm{H} / \mathrm{H}_{2}$ system over a wide range of parameter space. We show that good approximations can be established. Section 6 concludes the paper and contains a consideration of the physical conditions in star forming regions where the simple approximations used in Sects. 3 and 4 should give reliable results.

\section{An exact analytical treatment of a model of the pure hydrogen system}

Under conditions where reactions are second order, but only a very small number of particles are able to react, the classical deterministic nonlinear rate laws are well known to break down. Under these conditions it is necessary to characterise the system using a probability distribution for the numbers of particles of each species present and to deduce the expectation kinetics from the rates of transition between the possible states of the system. Stochastic kinetics, and in particular methods for solving the master equation, have been extensively reviewed by McQuarrie (1967), and has been applied in many areas.

In a simple grain model in which only hydrogen atoms and molecules are present in the gas phase and only $\mathrm{H}_{2}$ (assumed to be unreactive) is produced by surface chemistry, $P(i)$ is the probability that a grain contains $i$ hydrogen atoms. The master equation governing the system is

$$
\begin{aligned}
\frac{\mathrm{d} P(i)}{\mathrm{d} t}= & a_{\mathrm{H}}[P(i-1)-P(i)]+d_{\mathrm{H}}[(i+1) P(i+1)-i P(i)] \\
& +\frac{1}{2} k_{\mathrm{H}, \mathrm{H}}[(i+2)(i+1) P(i+2)-i(i-1) P(i)]
\end{aligned}
$$

where $a_{\mathrm{H}}$ is the rate of accretion, $d_{\mathrm{H}}$ and $k_{\mathrm{H}, \mathrm{H}}$ the rate coefficients for desorption and reaction on the grain. We suppose that steady state equilibrium pertains.

We define the generating function

$$
g(s) \equiv \sum_{i=0}^{\infty} P(i) s^{i}
$$

Use of (1) and (2) and $\mathrm{d} P(i) / \mathrm{d} t=0$ gives

$\frac{1}{2} k_{\mathrm{H}, \mathrm{H}}\left(1-s^{2}\right) \frac{\mathrm{d}^{2} g}{\mathrm{~d} s^{2}}+d_{\mathrm{H}}(1-s) \frac{\mathrm{d} g}{\mathrm{~d} s}-a_{\mathrm{H}}(1-s) g=0$.

(3) The probability of state $i$ can be found from the generating

Defining

$r \equiv 1+s$

(4) $P(i)=\frac{g^{(i)}(s=0)}{i !}$.

Hence, the solution is not differentiable at $r=0$ unless the constant $A$ is zero. Thus, we find that

$g(s)=B(1+s)^{(1-2 \delta) / 2} I_{-(1-2 \delta)}(\sqrt{8 \alpha(1+s)})$.

The constant $B$ can be found from normalisation, since $g(s=1)=1$ as seen from Eq. (2) and the requirement that the probabilities sum to unity. Hence,

$g(s)=\left(\frac{1+s}{2}\right)^{(1-2 \delta) / 2} \frac{I_{-(1-2 \delta)}(\sqrt{8 \alpha(1+s)})}{I_{-(1-2 \delta)}(\sqrt{16 \alpha})}$.

function by differentiation: 
It is possible to find a general form for the derivatives of the generating function. Substituting $z=\sqrt{8 \alpha(1+s)}$ we find

$$
\begin{aligned}
g^{(i)}= & \frac{2^{2(i-(1-2 \delta))} \alpha^{(i-(1-2 \delta) / 2)}}{I_{-(1-2 \delta)}(\sqrt{16 \alpha})}\left(\frac{1}{z} \frac{\mathrm{d}}{\mathrm{d} z}\right)^{i}\left(z^{\nu} I_{-(1-2 \delta)}(z)\right) \\
= & \left(\frac{1+s}{2}\right)^{(1-2 \delta) / 2}\left(\frac{2 \alpha}{1+s}\right)^{i / 2} \\
& \times \frac{I_{i-(1-2 \delta)}(\sqrt{8 \alpha(1+s)})}{I_{-(1-2 \delta)}(\sqrt{16 \alpha})}
\end{aligned}
$$

which gives explicit solutions for the occupation probability of state $i$

$P(i)=\frac{g^{(i)}(0)}{i !}=\left(\frac{1}{2}\right)^{(1-2 \delta) / 2} \frac{(2 \alpha)^{i / 2}}{i !} \frac{I_{i+2 \delta-1}(\sqrt{8 \alpha})}{I_{2 \delta-1}(\sqrt{16 \alpha})}$.

The formation rate of $\mathrm{H}_{2}$ is

$\Gamma\left(\mathrm{H}_{2}\right)=\frac{k_{\mathrm{H}, \mathrm{H}}}{2} \sum_{i=0}^{\infty} i(i-1) P(i)=\frac{k_{\mathrm{H}, \mathrm{H}}}{2}<i(i-1)>$.

This equation points out the two basic differences between a stochastic solution and a deterministic solution. Firstly the number of pairs on the surface is $i(i-1) / 2$. Secondly note the correct position of the expectation. In deterministic kinetics the rate is assumed proportional to $\langle i\rangle^{2}$. The deterministic equation therefore ignores the $-i$, which is extremely important if the number of particles present is very small, as on a typical grain surface. In addition, it assumes $\left.<i^{2}\right\rangle=\langle i\rangle^{2}$, which is equivalent to neglecting statistical fluctuations in the number of particles, and is the reason for describing the classical rate law as deterministic. From Eqs. (2) and (20) we find that

$\Gamma\left(\mathrm{H}_{2}\right)=\frac{k_{\mathrm{H}, \mathrm{H}}}{2} \frac{\mathrm{d}^{2} g(s=1)}{\mathrm{d} s^{2}}$.

Use of (18) in (21) gives

$\Gamma\left(\mathrm{H}_{2}\right)=\Gamma^{\mathrm{E}}\left(\mathrm{H}_{2}\right) \equiv \frac{k_{\mathrm{H}, \mathrm{H}}}{2} \alpha \frac{I_{1+2 \delta}(\sqrt{16 \alpha})}{I_{-1+2 \delta}(\sqrt{16 \alpha})}$

an exact result.

The model's validity is subject to a number of restrictions. One is that $a_{\mathrm{H}}$ varies only on a timescale very long compared to that for equilibrium on the surfaces to be approached. If $\left\langle i>\leq 1, a_{\mathrm{H}}^{-1}\right.$ is a rough upper bound to the timescale for equilibrium to be reached on a grain surface; if $\langle i\rangle \gg 1$, the deterministic rate equation can be used to give an estimate for $\langle i\rangle$ and the timescale for equilibrium on a grain surface to be reached is found to be approximately $a_{\mathrm{H}}^{-1}\left[-\delta+\left(\delta^{2}+4 \alpha\right)^{1 / 2}\right] / 2$. Juna (1974) argued that $\mathrm{H}_{2}$ and $\mathrm{H}$ data for diffuse interstellar clouds are in harmony with the gas phase $\mathrm{H}_{2}$ formation rate per unit volume being of order $10^{-17} \mathrm{~cm}^{-3} \mathrm{~s}^{-1}\left(n_{\mathrm{H}} n(\mathrm{H}) / \mathrm{cm}^{-6}\right)$ at $10 \mathrm{~K}$, with $n_{\mathrm{H}}$ and $n(\mathrm{H})$ being the gas phase hydrogen nucleus and atomic hydrogen number densities. For $n_{\mathrm{H}}=10^{5} \mathrm{~cm}^{-3}$, which is typical for a dark region in a dense core (cf. Myers 1990), and $n(\mathrm{H})=1 \mathrm{~cm}^{-3}$, and
$a_{\mathrm{H}}=1.45 \times 10^{-5} \mathrm{~s}^{-1}\left(n(\mathrm{H}) / \mathrm{cm}^{-3}\right)$ (e.g. Caselli et al. 1998), the ratio of the timescale on which $n(\mathrm{H})$ and $a_{\mathrm{H}}$ change to $a_{\mathrm{H}}^{-1}$ is of the order of $10^{7}$. As long as $\left[-\delta+\left(\delta^{2}+4 \alpha\right)^{1 / 2}\right] / 2$ is much smaller than this large quantity, the assumption of a constant value of $a_{\mathrm{H}}$ is reasonable.

Another restriction is that the number of hydrogen atoms on a typical grain is much smaller than the number of reaction sites on it. That latter number has been suggested to be of the order of $10^{6}$ (e.g. Tielens \& Allamandola 1987). As long as $\left[-\delta+\left(\delta^{2}+4 \alpha\right)^{1 / 2}\right] / 2$ is much smaller than this large number and it is a reasonable estimate for the number of reaction sites the restriction is unimportant.

Biham et al. (2001) have also examined the $\mathrm{H} / \mathrm{H}_{2}$ system using numerical solutions of the master equation. They incorporated desorption of $\mathrm{H}_{2}$ which is not included explicitly in the treatment given above. The only reaction taking place on the surface is $\mathrm{H}+\mathrm{H}$ the desorption of $\mathrm{H}_{2}$ is irrelevant to the steady-state rate of $\mathrm{H}_{2}$ production and so our analysis is unaffected by it. If $\mathrm{H}_{2}$ also reaches a steady-state on the grain then the rate of desorption is equal to the rate of production, $\Gamma\left(\mathrm{H}_{2}\right)$.

\section{The method applied to the hydrogen and oxygen system}

To extend the method to more complex systems we focus on the $\mathrm{H}, \mathrm{H}_{2}, \mathrm{O}, \mathrm{OH}$, and $\mathrm{O}_{2}$ problem addressed by Caselli et al. (1998), in which reactions are limited to

$\mathrm{H}+\mathrm{H} \rightarrow \mathrm{H}_{2}$

$\mathrm{O}+\mathrm{H} \rightarrow \mathrm{OH}$

$\mathrm{O}+\mathrm{O} \rightarrow \mathrm{O}_{2}$.

The "exact" production rates of $\mathrm{H}_{2}, \mathrm{OH}$, and $\mathrm{O}_{2}$ are

$\Gamma\left(\mathrm{H}_{2}\right)=\frac{k_{\mathrm{H}, \mathrm{H}}}{2} \sum_{\substack{i=2 \\ j=0}}^{\infty} P(i, j)(i)(i-1)$,

$\Gamma\left(\mathrm{O}_{2}\right)=\frac{k_{\mathrm{O}, \mathrm{O}}}{2} \sum_{\substack{i=0 \\ j=2}}^{\infty} P(i, j)(j)(j-1)$,

$\Gamma(\mathrm{OH})=k_{\mathrm{O}, \mathrm{H}} \sum_{\substack{i=1 \\ j=1}}^{\infty} P(i, j)(i)(j)$,

where $P(i, j)$ is the probability that a grain surface contains $i$ hydrogen atoms and $j$ oxygen atoms.

The $P(i, j)$ s form the solution to a master equation which, for the simple system under investigation, is

$$
\begin{aligned}
& \frac{\mathrm{d} P(i, j)}{\mathrm{d} t}=a_{\mathrm{H}}[P(i-1, j)-P(i, j)]+a_{\mathrm{O}}[P(i, j-1) \\
& -P(i, j)]+d_{\mathrm{H}}[P(i+1, j)(i+1)-i P(i, j)] \\
& -\frac{k_{\mathrm{H}, \mathrm{H}}}{2}[P(i, j)(i)(i-1)-P(i+2, j)(i+2)(i+1)] \\
& -\frac{k_{\mathrm{O}, \mathrm{O}}}{2}[P(i, j)(j)(j-1)-P(i, j+2)(j+2)(j+1)] \\
& -k_{\mathrm{O}, \mathrm{H}}[P(i, j)(i)(j)-P(i+1, j+1)(i+1)(j+1)],
\end{aligned}
$$


Table 1. Rates and rate coefficients in $\mathrm{s}^{-1}$ used in the $\mathrm{H}, \mathrm{O}$ and H, N, O systems (Caselli et al. 1998).

\begin{tabular}{rr|ll}
\hline$k_{\mathrm{H}, \mathrm{H}}$ & $1.02(+05)$ & $a_{\mathrm{H}}$ & $1.45(-05) \times\left(n(\mathrm{H}) / \mathrm{cm}^{-3}\right)$ \\
$k_{\mathrm{O}, \mathrm{H}}$ & $5.1(+04)$ & $a_{\mathrm{O}}$ & $3.6(-06) \times\left(n(\mathrm{O}) / \mathrm{cm}^{-3}\right)$ \\
$k_{\mathrm{O}, \mathrm{O}}$ & $8.4(-05)$ & $a_{\mathrm{N}}$ & $3.9(-06) \times\left(n(\mathrm{~N}) / \mathrm{cm}^{-3}\right)$ \\
$k_{\mathrm{N}, \mathrm{O}}$ & $8.7(-05)$ & & \\
$k_{\mathrm{N}, \mathrm{N}}$ & $9.0(-05)$ & & \\
$d_{\mathrm{H}}$ & $1.9(-03)$ & & \\
\hline
\end{tabular}

The accretion rate of a species $m, a_{m}$, depends on the gas-phase number density of the species, $n(m)$, and refers to accretion on to an average grain at $10 \mathrm{~K}$. The notation $a(b)$ implies $a \times 10^{b}$.

where $a_{\mathrm{H}}$ and $a_{\mathrm{O}}$ are the rates at which atomic hydrogen and atomic oxygen are accreted from the gas phase, and $d_{\mathrm{H}}$ is the rate coefficient for atomic hydrogen desorption. Desorption of $\mathrm{O}$ is assumed negligible. Values of the rates and rate coefficients are presented in Table 1 (cf. Caselli et al. 1998). In this paper we neglect time derivatives as gas phase chemical composition changes on much longer timescales than those relevant to the active surface chemistry.

Solutions to the master equation for large truncation values of $i, j$ were obtained through the use of the row-indexed method for the inversion of a sparse matrix. Examination of the results under the conditions of interest shows that the $P(i, j)$ s decrease very rapidly as $i$ and $j$ are increased. Since the master equation becomes increasingly complex as additional chemical species are introduced it is useful to consider approximations to the master equation approach.

Though a lower value of $k_{\mathrm{H}, \mathrm{H}}$ has been advocated (Katz et al. 1999) we use the values of $k_{\mathrm{H}, \mathrm{H}}$ and $k_{\mathrm{O}, \mathrm{H}}$ adopted by Caselli et al. (1998). The physical chemical microprocesses regulating the formation of $\mathrm{H}_{2}$ on grain surfaces are currently the subject of considerable theoretical and experimental investigation aimed at the removal of the huge uncertaintly in $k_{\mathrm{H}, \mathrm{H}}$; Charnley (2001) has stressed that the kinetic calculations based on the results of such investigation yield values of $k_{\mathrm{H}, \mathrm{H}}$ and other rate coefficients that depend extremely sensitively on the assumed dimensionality of the grain surfaces, and that twodimensional rather than one-dimensional diffusion theory must be used if the grain surfaces are two dimensional. Because $k_{\mathrm{H}, \mathrm{H}}$ is so large, we can develop analytic approximations by assuming that on any grain that already contains a reactive species, a reaction occurs instantaneously when a new $\mathrm{H}$ atom is accreted. Thus, we may take all $P(i, j)$ s except $P(0,0), P(0,1)$, and $P(1,0)$ to be small. The values given for $P(i, j)$ by the approximate method will be designated $P^{\mathrm{A}}(i, j)$. Our basic assumption leads us to take

$P^{\mathrm{A}}(0,0)+P^{\mathrm{A}}(0,1)+P^{\mathrm{A}}(1,0)=1$.
Consistent with our main assumption, we take

$\Gamma\left(\mathrm{H}_{2}\right) \approx \Gamma^{\mathrm{A}}\left(\mathrm{H}_{2}\right) \equiv a_{\mathrm{H}} P^{\mathrm{A}}(1,0)$.

For similar reasons we use

$\Gamma(\mathrm{OH}) \approx \Gamma^{\mathrm{A}}(\mathrm{OH}) \equiv a_{\mathrm{O}} P^{\mathrm{A}}(1,0)+a_{\mathrm{H}} P^{\mathrm{A}}(0,1)$.

Because the value of $k_{\mathrm{O}, \mathrm{O}}$ is markedly less than the values adopted for $k_{\mathrm{H}, \mathrm{H}}$ and $k_{\mathrm{O}, \mathrm{H}}$, our approximation to $\Gamma\left(\mathrm{O}_{2}\right)$ is somewhat different, in order to account for the possibility that one (or both) of the $\mathrm{O}$ atoms may react with an accreting $\mathrm{H}$ before $\mathrm{O}_{2}$ can be formed. It is

$\Gamma\left(\mathrm{O}_{2}\right) \approx \Gamma^{\mathrm{A}}\left(\mathrm{O}_{2}\right) \equiv k_{\mathrm{O}, \mathrm{O}} P^{\mathrm{A}}(0,2)$

where

$P^{\mathrm{A}}(0,2)=\frac{a_{\mathrm{O}}}{a_{\mathrm{H}}+a_{\mathrm{O}}+k_{\mathrm{O}, \mathrm{O}}} P^{\mathrm{A}}(0,1)$.

Substituting $P^{\mathrm{A}}(0,0), P^{\mathrm{A}}(0,1)$, and $P^{\mathrm{A}}(1,0)$ for $P(0,0)$, $P(0,1)$, and $P(1,0)$ in Eq. $(7)$, taking all other $P(i, j)$ 's to be negligible, and using Eqs. (30) through (34), we find

$$
P^{\mathrm{A}}(0,0)=\left\{1+\frac{a_{\mathrm{H}}}{a_{\mathrm{H}}+a_{\mathrm{O}}+d_{\mathrm{H}}}+\frac{a_{\mathrm{O}}}{a_{\mathrm{H}}+a_{\mathrm{O}}}\right\}^{-1}
$$

and

$$
\begin{aligned}
& \Gamma^{\mathrm{A}}\left(\mathrm{H}_{2}\right)=\frac{a_{\mathrm{H}}^{2} P^{\mathrm{A}}(0,0)}{a_{\mathrm{H}}+a_{\mathrm{O}}+d_{\mathrm{H}}} \\
& \Gamma^{\mathrm{A}}(\mathrm{OH})=a_{\mathrm{O}} a_{\mathrm{H}}\left[\frac{1}{a_{\mathrm{H}}+a_{\mathrm{O}}+d_{\mathrm{H}}}+\frac{1}{a_{\mathrm{O}}+a_{\mathrm{H}}}\right] P^{\mathrm{A}}(0,0) \\
& \Gamma^{\mathrm{A}}\left(\mathrm{O}_{2}\right)=\frac{k_{\mathrm{O}, \mathrm{O}}}{k_{\mathrm{O}, \mathrm{O}}+a_{\mathrm{O}}+a_{\mathrm{H}}} \frac{a_{\mathrm{O}}^{2}}{a_{\mathrm{O}}+a_{\mathrm{H}}} P^{\mathrm{A}}(0,0)
\end{aligned}
$$

Figures 1 and 2 in Caselli et al. contain their results for the system we are considering in this section. We took values of all rates and rate coefficients to be identical to those used by Caselli et al. in their standard rate equation calculations (cf. Table 1), and as they did, we took the number density of gas phase atomic oxygen, $n(\mathrm{O})$, to be $1 \mathrm{~cm}^{-3}$ and let the value of the gas phase number density of atomic hydrogen, $n(\mathrm{H})$, vary.

Figure 1 shows results for the mole fractions of $\mathrm{H}_{2}$, $\mathrm{OH}$, and $\mathrm{O}_{2}$ given by the use of the approximate formation rates in Eqs. (36) to (38) and by the rate equations for the various values of $n(\mathrm{H})$, and for the fixed value of $n(\mathrm{O})=1 \mathrm{~cm}^{-3}$. The lower half of that figure gives the percentage differences between the approximate mole fractions and the mole fractions obtained from the master equation approach (Eqs. (26)-(29)) under the assumption that $P(i, j)=0$ if $i$ or $j>2$. The upper half of Fig. 2 shows that the truncation at higher values of $i, j$ gives only slightly more accurate values for the formation rates than truncation at $i, j=2,2$ does. The lower half of Fig. 2 shows that values of the formation rates given by the approximation are within a few percent of the accurate values. Indeed, when we plotted accurate values of the mole fractions on the same figure as the approximate values, 


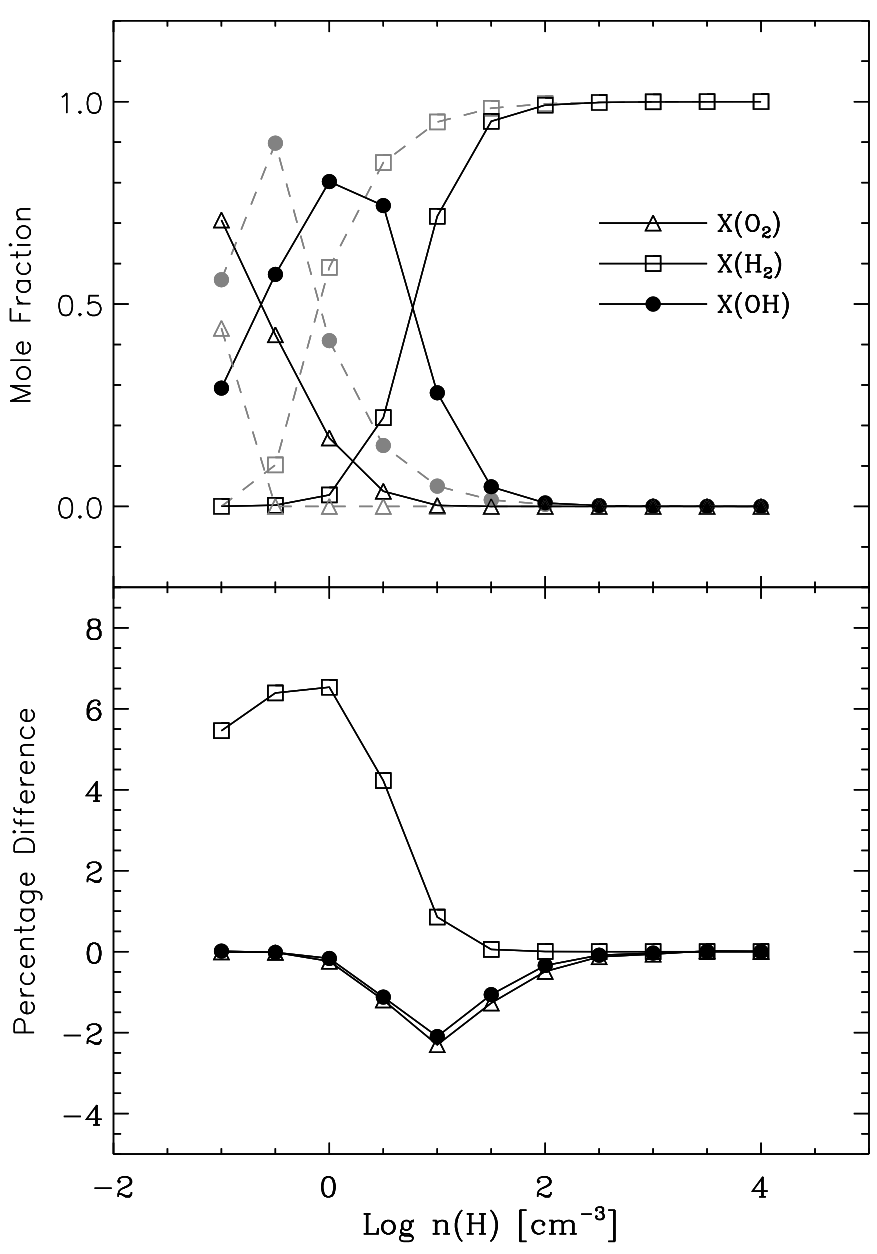

Fig. 1. Mole fractions of $\mathrm{H}_{2}, \mathrm{OH}$, and $\mathrm{O}_{2}$. The upper panel shows results for the mole fractions $(\mathrm{X})$ as functions of $n(\mathrm{H})$ for $n(\mathrm{O})=1 \mathrm{~cm}^{-3}$. Solid curves are the results given by the use of Eqs. (36) to (38), while the lighter, broken curves are the results given by the standard deterministic rate equation approach. The lower panel shows the percentage differences as functions of $n(\mathrm{H})$ between results given by the use of Eqs. (36) to (38) with $P(i, j)$ 's calculated from Eqs. (26)-(29) on the assumption that $P(i, j)=0$ if either $i>2$ or $j>2$.

we had difficulty visually distinguishing the "accurate" curves from the "approximate" curves.

Comparison of the results in Figs. 1 and 2 with the Monte Carlo results given by Caselli et al. (1998) in their Fig. 1 shows discrepancies. However, we have found our results to be in good agreement with those of revised Monte Carlo calculations (Herbst \& Stantcheva 2000). Caselli et al. (1998) introduced three modifications, and only the first brings rate equation results closer to our results.

Our results are similar to those of Allen \& Robinson (1977) (cf. Eqs. (36)-(38) with their Eq. (6)). However, we include $\mathrm{H}$ atom desorption and use a correction term for species formed via slow surface reactions (e.g. Eqs. (33)(34)). The Allen \& Robinson results are only applicable for the case of two reactive species on the surface, whereas our approach allows truncation at higher grain populations, permitting investigation into the errors involved in applying different cutoffs to the surface population (e.g.

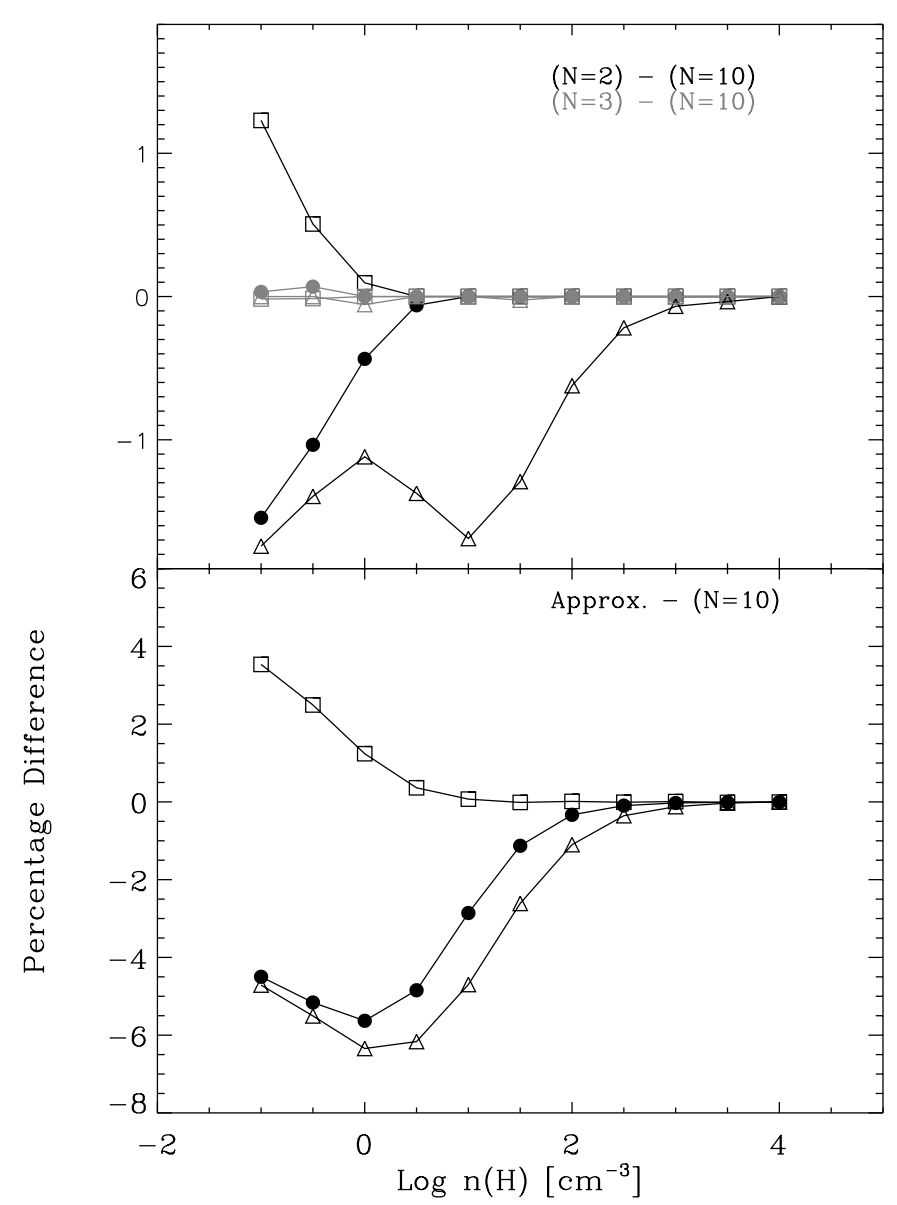

Fig. 2. Comparison of absolute values of $\mathrm{H}_{2}, \mathrm{OH}$, and $\mathrm{O}_{2}$ formation rates. The upper panel shows the percentage differences between values of the formation rates calculated with the use of Eqs. (26) to (29) on the assumption that $P(i, j)=0$ if either $i>N$ or $j>N$. Values of $N=2,3$ and 10 were considered. The lower panel displays percentage differences between results obtained with Eqs. (36) to (38) and the $N=10$ truncation results. As in Fig. 1, the squares, circles, and triangles indicate results for $\mathrm{H}_{2}, \mathrm{OH}$, and $\mathrm{O}_{2}$ respectively.

Fig. 2). We note that the similarity in expressions, coupled with the fact that the production rates used are almost identical, leads to the results obtained here (for the case of truncation at $N=2$, which implies $i, j=2,2)$ being approximately the same as those of Allen \& Robinson (1977).

\section{The hydrogen, nitrogen, and oxygen system}

Caselli et al. applied their modified deterministic rate equation approach to a simple chemical network involving hydrogen, nitrogen, and oxygen. We now turn to this network, the details of which are described by Caselli et al. Table 2 describes the reactions contained in the network. We constructed a master equation, analogous to Eq. (29), governing the distribution function giving the probability that a grain surface contains given numbers of $\mathrm{H}, \mathrm{N}$, and $\mathrm{O}$ atoms and $\mathrm{OH}, \mathrm{O}_{2}, \mathrm{H}_{2} \mathrm{O}, \mathrm{NH}, \mathrm{NH}_{2}, \mathrm{NH}_{3}, \mathrm{~N}_{2}$, and NO molecules. 
Table 2. H, N, and O reaction network.

\begin{tabular}{lll}
\hline $\mathrm{N}+\mathrm{H}$ & $\rightarrow$ & $\mathrm{NH}$ \\
$\mathrm{N}+\mathrm{N}$ & $\rightarrow$ & $\mathrm{N}_{2}$ \\
$\mathrm{~N}+\mathrm{O}$ & $\rightarrow$ & $\mathrm{NO}$ \\
$\mathrm{H}+\mathrm{NH}$ & $\rightarrow$ & $\mathrm{NH}_{2}$ \\
$\mathrm{H}+\mathrm{OH}$ & $\rightarrow$ & $\mathrm{H}_{2} \mathrm{O}$ \\
$\mathrm{H}+\mathrm{NH}_{2}$ & $\rightarrow$ & $\mathrm{NH}_{3}$ \\
\hline
\end{tabular}

Note: Eqs. (23)-(25) are also included in the chemical network.
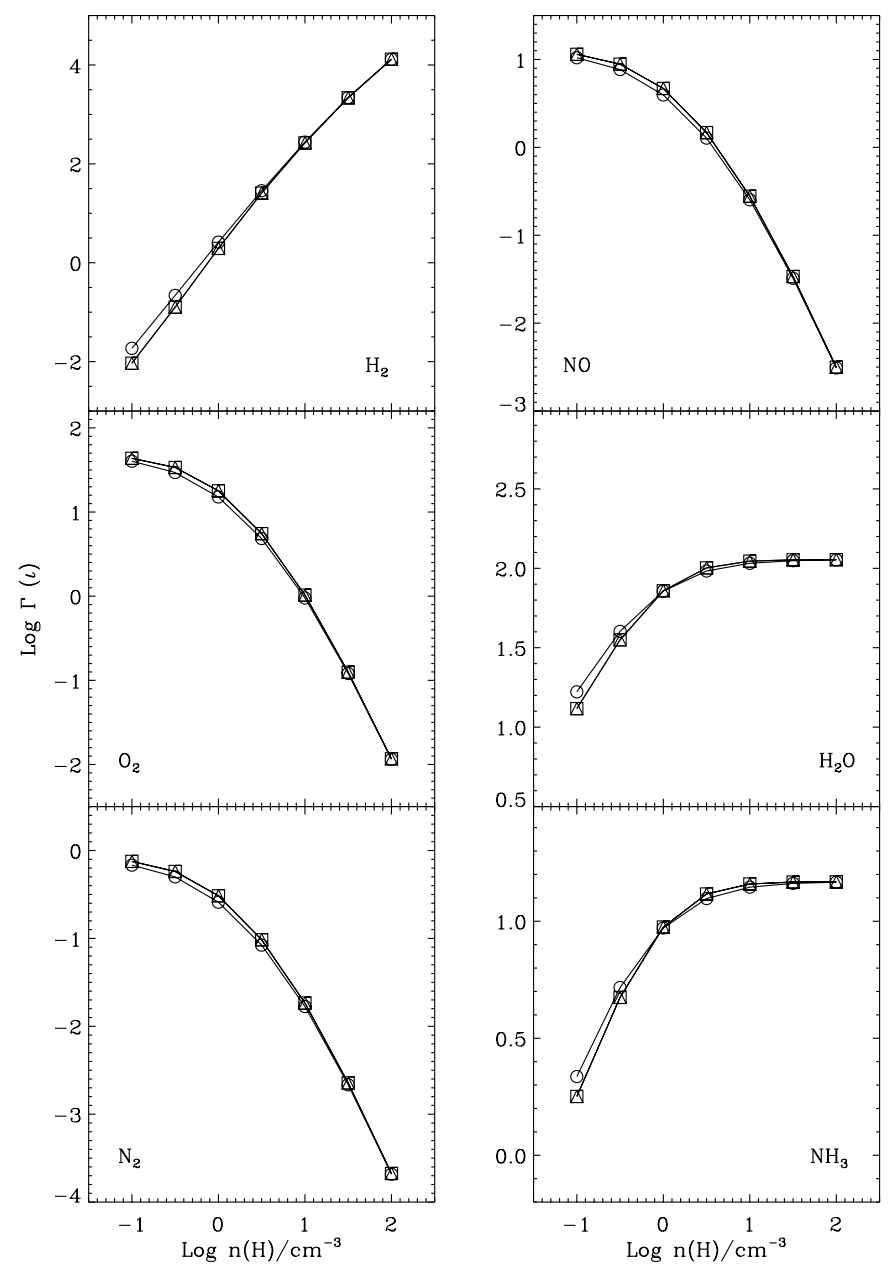

Fig. 3. Formation rates in the hydrogen, nitrogen, oxygen chemistry. $\Gamma(i)$, the formation rate per year of species $i$, is given for each of several species as a function of $n(\mathrm{H}) \cdot n(\mathrm{O})$ and $n(\mathrm{~N})$ were taken to be $1 \mathrm{~cm}^{-3}$ and $0.12 \mathrm{~cm}^{-3}$ respectively. Triangles, squares, and circles indicate results from a $N=8$ truncated solution to the master equation, $N=2$ truncated solution to the master equation, and the approximate treatment respectively.

This simple mechanism, with $\mathrm{NH}$ and $\mathrm{NH}_{2}$ reacting to form only $\mathrm{NH}_{2}$ and $\mathrm{NH}_{3}$ respectively, results in equal rates of formation of $\mathrm{NH}, \mathrm{NH}_{2}$ and $\mathrm{NH}_{3}$ in the steady-state; similar considerations apply to $\mathrm{OH}$ and $\mathrm{H}_{2} \mathrm{O}$. Approximations for the formation rates of various species, analogous to those of Eqs. (36) to (38), are easily constructed.
The approximations to the formation rates of $\mathrm{H}_{2}, \mathrm{OH}$, and $\mathrm{O}_{2}$ are obtained from these equations through the replacement of $a_{\mathrm{H}}+a_{\mathrm{O}}$ with $a$, where

$a \equiv a_{\mathrm{H}}+a_{\mathrm{O}}+a_{\mathrm{N}}$,

and $a_{\mathrm{N}}$ is the accretion rate of atomic nitrogen. Below, $k_{\mathrm{N}, \mathrm{O}}$ and $k_{\mathrm{N}, \mathrm{N}}$ are the standard deterministic surface rate coefficients for

$\mathrm{N}+\mathrm{O} \rightarrow \mathrm{NO}$

$\mathrm{N}+\mathrm{N} \rightarrow \mathrm{N}_{2}$.

Equation (35) is replaced with

$P^{\mathrm{A}}(0,0,0)=\left\{1+\frac{a_{\mathrm{H}}}{a+d_{\mathrm{H}}}+\frac{a_{\mathrm{O}}+a_{\mathrm{N}}}{a}\right\}^{-1}$,

where the three indices refer to the number of $\mathrm{H}, \mathrm{N}$, and $\mathrm{O}$ atoms on the grain. Approximate values of the formation rates of $\mathrm{NH}_{3}, \mathrm{~N}_{2}$, and $\mathrm{NO}$ are

$$
\begin{aligned}
& \Gamma^{\mathrm{A}}\left(\mathrm{NH}_{3}\right)=a_{\mathrm{N}} a_{\mathrm{H}}\left[\frac{1}{a+d_{\mathrm{H}}}+\frac{1}{a}\right] P^{\mathrm{A}}(0,0,0) \\
& \Gamma^{\mathrm{A}}\left(\mathrm{N}_{2}\right)=\frac{k_{\mathrm{N}, \mathrm{N}}}{k_{\mathrm{N}, \mathrm{N}}+a} \frac{a_{\mathrm{N}}^{2}}{a} P^{\mathrm{A}}(0,0,0) \\
& \Gamma^{\mathrm{A}}(\mathrm{NO})=\frac{2 k_{\mathrm{N}, \mathrm{O}}}{k_{\mathrm{N}, \mathrm{O}}+a} \frac{a_{\mathrm{N}} a_{\mathrm{O}}}{a} P^{\mathrm{A}}(0,0,0) .
\end{aligned}
$$

Figures 3 and 4 show results for the formation rates and differences between them obtained through the evaluation of the $\Gamma^{\mathrm{A}} \mathrm{S}$ and the use of the truncation at $N=2$ and $N=8$ in the solution of the master equation. Comparison of the results in Fig. 3 with those in Fig. 3 of Caselli et al. show that, as they expected, the Monte Carlo method is unreliable for the calculation of the $\mathrm{H}_{2}$ formation rate where that rate is small; also their modified rate equations give a very inaccurate $\mathrm{H}_{2}$ formation rate under such circumstances. Typically, agreement between our accurate results and our approximate results for this chemical system appears rather better than that between the Monte Carlo and modified rate equation results of Caselli et al. for other rates. As log-log plots have been used, the accuracy of the Caselli et al. Monte Carlo results for the rates of formation of species other than $\mathrm{H}_{2}$ cannot be assessed through comparison with our $N=8$ truncation results.

Except for the $\mathrm{H}_{2}$ formation rate at low values of $n(\mathrm{H})$, our approximate results are generally in good agreement with the accurate $N=8$ truncation results. At low values of $n(\mathrm{H})$ a significant fraction of grains contain one or two particles each of $\mathrm{OH}, \mathrm{NH}$, and $\mathrm{NH}_{2}$ as well as $\mathrm{O}$ and $\mathrm{N}$. Thus, in our approximations at low values of $n(\mathrm{H})$, we have overestimated the fraction of grains containing only one $\mathrm{H}$ atom, which leads to overestimates of the $\mathrm{H}_{2} \mathrm{O}$ and $\mathrm{NH}_{3}$ formation rates as well as the $\mathrm{H}_{2}$ formation rate. The overestimates of the $\mathrm{H}_{2} \mathrm{O}$ and $\mathrm{NH}_{3}$ formation rates are not as great as that of $\mathrm{H}_{2}$ because in our approximate treatment we have neglected the initiation of their formation on 

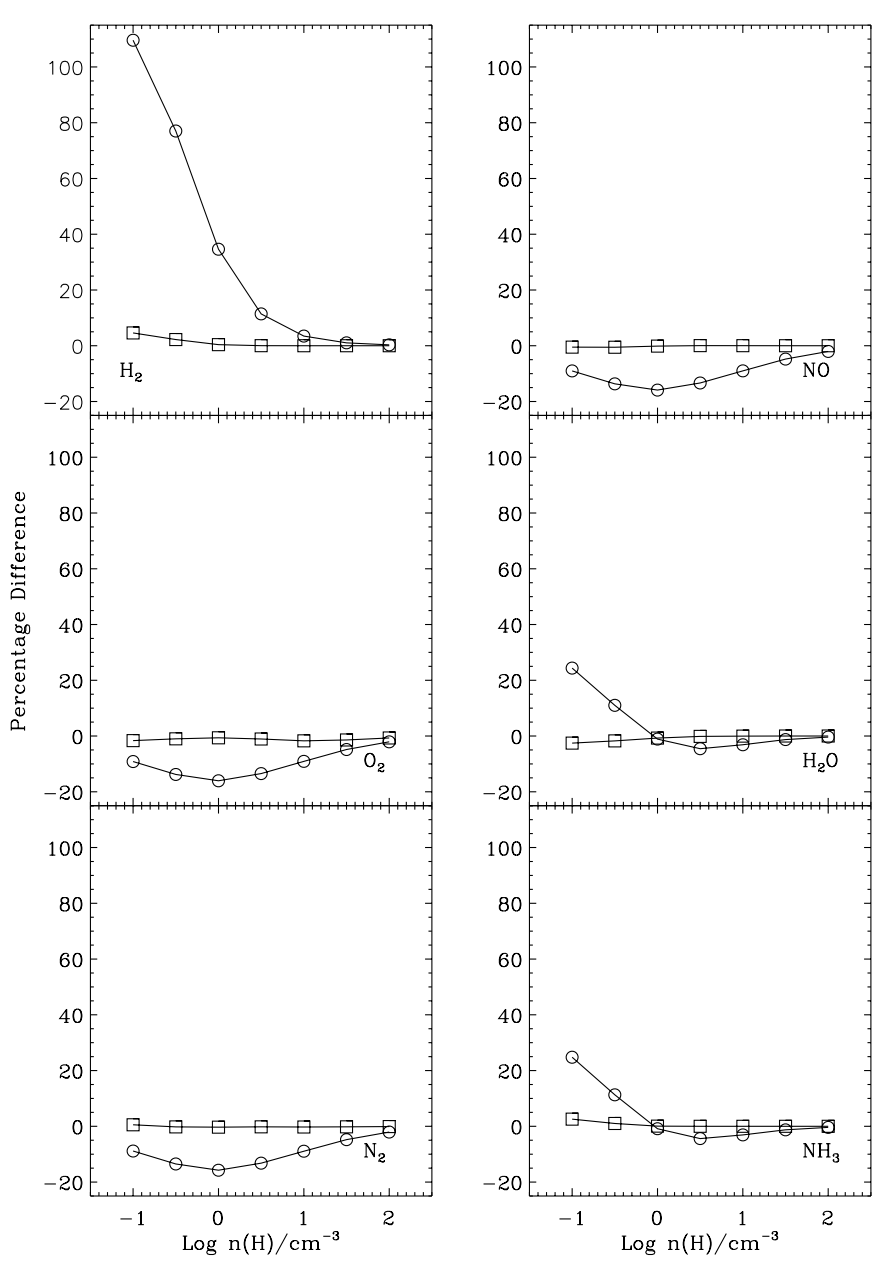

Fig. 4. Percentage differences of formation rates in the hydrogen, nitrogen, oxygen chemistry. Squares and circles indicate percentage differences between $N=2$ and $N=8$ truncation results and between the approximate and $N=8$ truncation results.

the highly populated grains mentioned just above. Neglect of $\mathrm{O}_{2}, \mathrm{~N}_{2}$, and $\mathrm{NO}$ formation on these highly populated grains leads to underestimates of their formation rates.

\section{Approximate versus exact results for the pure hydrogen system}

The analytic solution for the $\mathrm{H} / \mathrm{H}_{2}$ model, developed in Sect. 2 , is exact over the whole range of $\alpha\left(=a_{\mathrm{H}} / k_{\mathrm{H}, \mathrm{H}}\right)$ and $\delta\left(=d_{\mathrm{H}} / k_{\mathrm{H}, \mathrm{H}}\right)$ values and so provides a comparator for assessment of the performance of approximations.

We examine four approximations for the rate of formation of $\mathrm{H}_{2}$ :

(i) A deterministic model, based on the solution of standard chemical kinetic rate equations in the steady state, which gives:

$$
\Gamma^{\mathrm{d}}\left(\mathrm{H}_{2}\right) \equiv \frac{k_{\mathrm{H}, \mathrm{H}}}{8}\left[-\delta+\left(\delta^{2}+4 \alpha\right)^{1 / 2}\right]^{2} \text {. }
$$

(ii) The approximate stochastic model, based on the simplifications developed for the $\mathrm{O} / \mathrm{H}$ system in Sect. 3:

$\Gamma^{\mathrm{A}}\left(\mathrm{H}_{2}\right) \equiv k_{\mathrm{H}, \mathrm{H}} \frac{\alpha^{2}}{2 \alpha+\delta}$.

This result is based on the assumption that the rate is equal to $a_{\mathrm{H}} P(1)$, cf. Eq. (31).

(iii) $\Gamma^{\mathrm{A}}\left(\mathrm{H}_{2}\right)$ can be improved by including a factor $(1+\alpha) /(1+\alpha+\delta)$, which allows for desorption from a grain containing two $\mathrm{H}$ atoms competing with reaction or further desorption

$$
\Gamma^{\mathrm{ac}}\left(\mathrm{H}_{2}\right) \equiv\left(\frac{1+\alpha}{1+\alpha+\delta}\right) \Gamma^{\mathrm{A}}\left(\mathrm{H}_{2}\right) .
$$

(iv) Finally, a numerical solution of the master equation, $\Gamma^{2}\left(\mathrm{H}_{2}\right)$, was determined, on the assumption that no grain has more than two $\mathrm{H}$ atoms on its surface.

The percentage errors of the different approximations to $\Gamma\left(\mathrm{H}_{2}\right)$ are given by

$$
\begin{aligned}
& \Psi^{\mathrm{d}} \equiv 100 \frac{\left|\Gamma^{\mathrm{d}}\left(\mathrm{H}_{2}\right)-\Gamma^{\mathrm{E}}\left(\mathrm{H}_{2}\right)\right|}{\Gamma^{\mathrm{E}}\left(\mathrm{H}_{2}\right)} \\
& \Psi^{2} \equiv 100 \frac{\left|\Gamma^{2}\left(\mathrm{H}_{2}\right)-\Gamma^{\mathrm{E}}\left(\mathrm{H}_{2}\right)\right|}{\Gamma^{\mathrm{E}}\left(\mathrm{H}_{2}\right)} \\
& \Psi^{\mathrm{A}} \equiv 100 \frac{\left|\Gamma^{\mathrm{A}}\left(\mathrm{H}_{2}\right)-\Gamma^{\mathrm{E}}\left(\mathrm{H}_{2}\right)\right|}{\Gamma^{\mathrm{E}}\left(\mathrm{H}_{2}\right)} \\
& \Psi^{\mathrm{ac}} \equiv 100 \frac{\left|\Gamma^{\mathrm{ac}}\left(\mathrm{H}_{2}\right)-\Gamma^{\mathrm{E}}\left(\mathrm{H}_{2}\right)\right|}{\Gamma^{\mathrm{E}}\left(\mathrm{H}_{2}\right)} .
\end{aligned}
$$

Figure 5 contains contour plots of the functions $\Psi^{\mathrm{d}}, \Psi^{2}$, $\Psi^{\mathrm{A}}$, and $\Psi^{\mathrm{ac}}$ in $\alpha, \delta$ space.

$\Gamma^{\mathrm{d}}\left(\mathrm{H}_{2}\right), \Gamma^{\mathrm{A}}\left(\mathrm{H}_{2}\right)$, and $\Gamma^{\mathrm{ac}}\left(\mathrm{H}_{2}\right)$ are always larger than $\Gamma^{\mathrm{E}}\left(\mathrm{H}_{2}\right) . \Gamma^{\mathrm{ac}}\left(\mathrm{H}_{2}\right)$ is everywhere a better approximation to $\Gamma^{\mathrm{E}}\left(\mathrm{H}_{2}\right)$ than $\Gamma^{\mathrm{A}}\left(\mathrm{H}_{2}\right)$ is. $\Psi^{\text {best }}$ is the minimum of $\Psi^{\mathrm{ac}}$ and $\Psi^{\mathrm{d}}$, and a contour plot for $\Psi^{\text {best }}$ is shown in the lowest left hand panel of Fig. 5. The lowest right hand panel in Fig. 5 indicates where $\Psi^{\mathrm{ac}}<\Psi^{\mathrm{d}}$ and where $\Psi^{\mathrm{d}}<\Psi^{\mathrm{ac}}$. The maximum value of $\Psi^{\text {best }}$ is about 48.5 .

$\Gamma^{2}\left(\mathrm{H}_{2}\right)$ is larger than $\Gamma^{\mathrm{E}}\left(\mathrm{H}_{2}\right)$ in the upper left of the $\alpha-\delta$ plane and smaller in the lower right. Figure 5 shows clearly that in the regime where $\delta>\alpha$ the accuracy of $\Gamma^{2}\left(\mathrm{H}_{2}\right)$ is greater than that of $\Gamma^{\mathrm{ac}}\left(\mathrm{H}_{2}\right)$ and the accuracy of $\Gamma^{\mathrm{ac}}\left(\mathrm{H}_{2}\right)$ is far greater than that of $\Gamma^{\mathrm{A}}\left(\mathrm{H}_{2}\right)$. The accuracy of $\Gamma^{\mathrm{d}}\left(\mathrm{H}_{2}\right)$ is worst in the regime where $\delta>\alpha$ and $\delta<2$. Thus, of the estimates we have considered, $\Gamma^{2}\left(\mathrm{H}_{2}\right)$ provides the best agreement with $\Gamma^{\mathrm{E}}\left(\mathrm{H}_{2}\right)$ where the use of the standard deterministic rate equation approach is unreliable. Still, there is a large part of parameter space in which even our simplest estimate, $\Gamma^{\mathrm{A}}\left(\mathrm{H}_{2}\right)$, gives good agreement with $\Gamma^{\mathrm{E}}\left(\mathrm{H}_{2}\right)$.

Note that the range of $n_{\mathrm{H}}$ values covered in Sects. 3 and 4 , coupled with the rate parameters in Table 1 , correspond to $10^{-7} \leq \alpha \leq 10^{-1}$ and $\delta=1.9 \times 10^{-8}$. Katz et al. (1999) proposed a lower values for $k_{\mathrm{H}, \mathrm{H}}$. Taking $k_{\mathrm{H}, \mathrm{H}}=100 \mathrm{~s}^{-1}$ gives $10^{-4} \leq \alpha \leq 10^{-2}$ and $\delta=1.9 \times 10^{-5}$. 

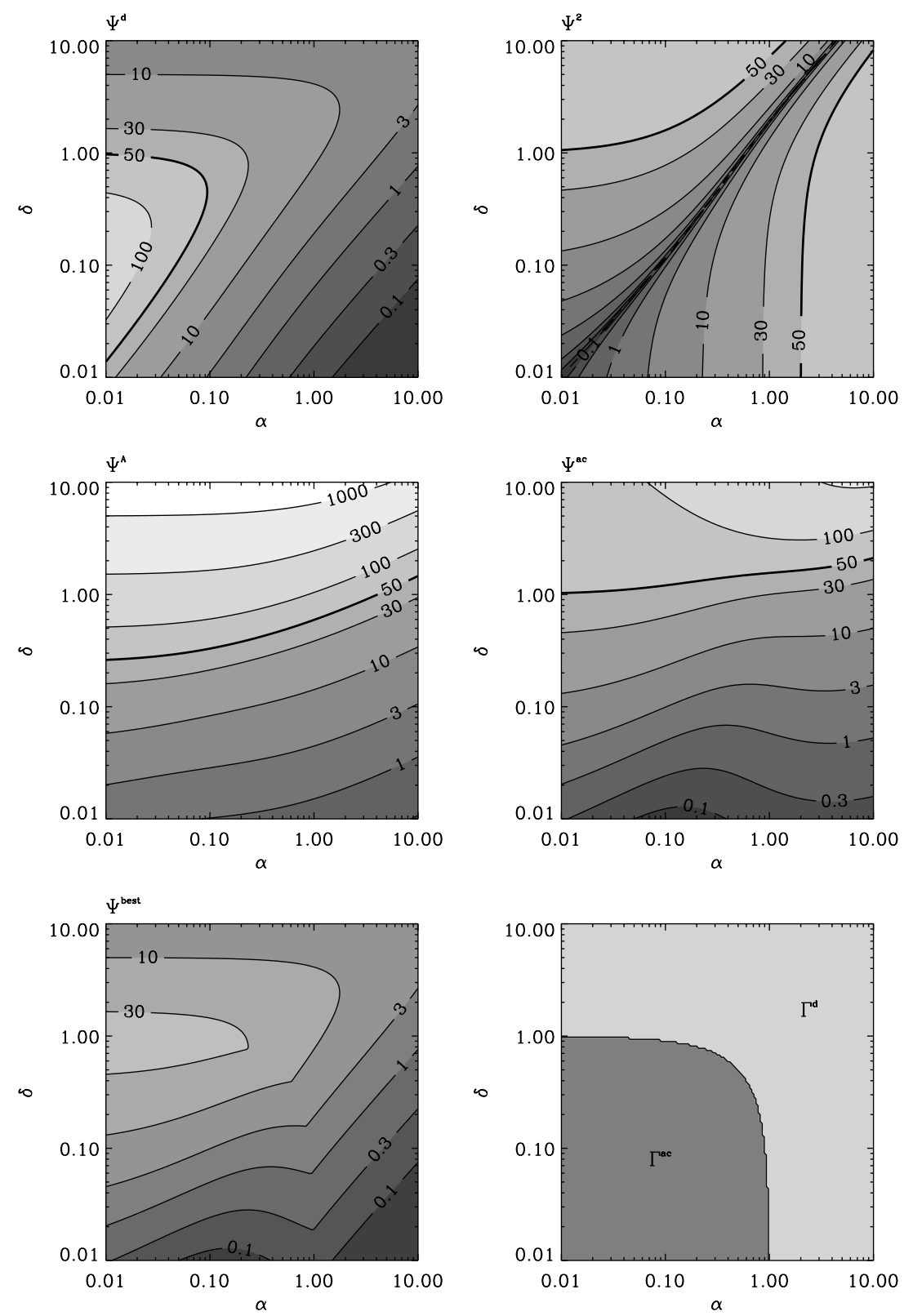

Fig. 5. Percentage errors of different approximations to the $\mathrm{H}_{2}$ formation rate in the pure hydrogen system. Except in the lowest right panel, darker regions are those in which the approximations are most accurate.

\section{Conclusions}

The master equation is simply an analytical formulation of the same probabilistic problem that others have studied with a Monte Carlo method. Using the same parameters the master equation approach and the Monte Carlo technique produce identical results. Yet for small systems the master equation method is much more computer efficient, and leads to the formulation of simplifying approximations.

The solution of the master equation on the assumption that no grain contains more than two of any given species yields accurate results for each of the hydrogen and oxygen and the hydrogen, nitrogen, and oxygen systems for the parameter regimes we investigated. The examination of the simple pure hydrogen system shows that the better of that approach and the standard deterministic rate equation approach gives an error of less than $50 \%$ in the $\mathrm{H}_{2}$ formation rate. Thus, a judicious combination of the two approaches should allow reasonably accurate solution of many grain surface chemical problems. The implementation of techniques for sparse matrix inversion would allow the treatment of much more complicated surface chemical networks with the $N=2$ truncation method than those we have considered.

We have introduced simple approximate expressions for the calculation of formation rates of species. They work well for the hydrogen and oxygen and the hydrogen, nitrogen, and oxygen systems for the parameter regimes we considered. However, the fractional (but not the absolute) error in the $\mathrm{H}_{2}$ formation rate is about $100 \%$ in the hydrogen, nitrogen, and oxygen system for the lowest values 
of the ratio of the atomic hydrogen and atomic oxygen accretion rates. Thus, the reliability of the use of simple approximate expressions like those we have introduced depends on $n(\mathrm{O})$ not exceeding $n(\mathrm{H})$.

We can reasonably doubt whether $n(\mathrm{O})$ is very often higher (in the sorts of cold regions for which the surface schemes are designed) than we have assumed it to be. $n(\mathrm{O}) \approx 1 \mathrm{~cm}^{-3}$ might be expected where the number density of hydrogen nuclei, $n_{\mathrm{H}}$, is about $10^{4} \mathrm{~cm}^{-3}$. It is well known that the depletion timescales at higher values of $n_{\mathrm{H}}$ are shorter than the dynamical timescales (e.g. Williams 1993), and the low fractional abundances of gas phase CO seen in a number of regions where $n_{\mathrm{H}}$ may be $\lesssim 10^{5} \mathrm{~cm}^{-3}$ (e.g. Gibb \& Little 1998; Willacy et al. 1998; Caselli et al. 1999; Kramer et al. 1999) indicate that depletion may often become important at low enough densities that $n(\mathrm{O})$ does not exceed about $1 \mathrm{~cm}^{-3}$. The failure to detect $\mathrm{O}_{2}$ and $\mathrm{H}_{2} \mathrm{O}$ features towards dense cores in low mass star forming regions (Goldsmith et al. 2000; Snell et al. 2000) provides further evidence of low gas phase abundances of elemental oxygen in many regions with $n_{\mathrm{H}}$ not hugely in excess of $10^{4} \mathrm{~cm}^{-3}$. In short, in many circumstances the surface chemistry may well have evolved far towards completion without $n(\mathrm{O})$ ever rising above $1 \mathrm{~cm}^{-3}$, which is the typical value of $n(\mathrm{H})$ in dark gas subjected to cosmic ray induced ionization at the standard rate of about $1 \times 10^{-17} \mathrm{~s}^{-1}$.

Acknowledgements. We wish to thank the referee, Dr. S. Charnley, for a careful reading of the paper which lead to many improvements.

\section{References}

Abramowitz, M., \& Stegun, I. A. (eds.) 1964, Handbook of Mathematical Functions, National Bureau of Standards, Washington DC
Biham, O., Furman, I., Pirronello, V., \& Vidali, G. 2001, ApJ, 553,595

Caselli, P., Hasegawa, T. I., \& Herbst, E. 1998, ApJ, 495, 309

Caselli, P., Walmsley, C. M., Tafalla, M., Dore, L., \& Myers, P. C. 1999, ApJ, 523, L165

Charnley, S. B. 2001, MNRAS, submitted

Charnley, S. B. 1998, ApJ, 509, L121

Charnley, S. B., Tielens, A. G. G. M., \& Rodgers, S. D. 1997, ApJ, 482, L203

Gibb, A. G., \& Little, L. T. 1998, MNRAS, 295, 299

Goldsmith, P. F., Melnick, G. J., Bergin, E. A., et al. 2000, ApJ, 539, L123

Herbst, E., \& Stantcheva, T. 2000, priv. commun.

Juna, M. 1974, ApJ, 191, 375

Katz, N., Furman, I., Biham, O., Pirronello, V., \& Vidali, G. 1999, ApJ, 522, 305

Kramer, C., Alves, J., Lada, C. J., et al. 1999, A\&A, 342, 257

McQuarrie, D. A. 1967, J. Appl. Prob., 4, 413

Myers, P. C. 1990, in Molecular Astrophysics - A Volume Honouring Alexander Dalgarno, ed. T. W. Hartquist (Cambridge University Press, Cambridge), 328

Ruffle, D. P., \& Herbst, E. 2000, MNRAS, 319, 837

Shalabiea, O. M., Caselli, P., \& Herbst, E. 1998, ApJ, 502, 652

Snell, R. L., Howe, J. E., Ashby, M. L. N., et al. 2000, ApJ, 539, L101

Tielens, A. G. G. M. 1995, unpublished

Tielens, A. G. G. M., \& Allamandola, L. J. 1987, in Interstellar Processes, ed. D. J. Hollenbach, \& H. A. Thronson (Reidel, Dordrecht), 397

Tielens, A. G. G. M., \& Charnley, S. B. 1997, Origins Life Evol. Biosphere, 27, 23

Tielens, A. G. G. M., \& Hagen, W. 1982, A\&A, 114, 245

Willacy, K., Langer, W. D., \& Velusamy, T. 1998, ApJ, 507, L171

Williams, D. A. 1993, in Dust and Chemistry in Astronomy, ed. T. J. Millar, \& D. A. Williams (Institute of Physics Printing, Bristol), 143 